\title{
Linear Matrix Inequality based static output feedback sliding mode control for discrete time systems
}

\author{
Srinath Govindaswamy, Thierry Floquet, and Sarah K. Spurgeon
}

\begin{abstract}
This paper discusses the design of a static output feedback based sliding mode controller for MIMO nonminimum phase discrete time systems. In previous work, it was shown that by identifying a minimal set of current and past outputs to determine an extended output signal, an augmented system can be obtained which permits the design of a sliding manifold based upon output information only, which renders the sliding mode dynamics stable. In this paper, it is shown that if the extended outputs chosen span the state zero directions of an invariant zero of the system, then the invariant zero disappears from the augmented system. Linear matrix inequalities are then used for sliding surface design. Such controllers have great potential within reconfigurable control schemes for application in the presence of multiple sensor failures. The methodology is applied to an aircraft system to show the efficacy of the design philosophy.
\end{abstract}

\section{INTRODUCTION}

In continuous time, a sliding mode is generated by means of discontinuities in the control signals about a surface in the state space [29]. It is required that the discontinuity surface, usually called the sliding surface, is attained from any initial condition in a finite time interval. For an appropriately selected controller, the motion on the surface, or sliding mode, is completely insensitive to any matched uncertainty in the system [29], [6]. In a discrete control implementation, the control signal is held constant during the sample period and hence it is not possible, in general, to attain a sliding mode which would require the control to switch at infinite frequency. As a result, the invariance properties of continuous time sliding-mode control can be lost. The obvious solution of sampling at high frequency, which will closely approximate continuous time, may not be possible for given hardware specifications. This has led to interest in the area of discrete time sliding-mode control (DSMC). For the case of uncertain discrete systems, it is not possible to ensure the states remain on a surface within the state space and for this reason much of the early DSMC literature focused on establishing a discrete time counterpart to the (continuous time) reachability condition [3], [11], [23]. A comprehensive overview of these early developments is given in [19]. One distinctive feature is that DSMC does not necessarily require the use of a discontinuous control strategy [26]. The results

Srinath Govindaswamy and Sarah Spurgeon are with the Instrumentation, Control \& Embedded Systems Research Group, Department of Electronics, University of Kent, Canterbury CT2 7NT, UK. S.K.Spurgeon@kent.ac.uk

Thierry Floquet is with LAGIS UMR CNRS 8146, École Centrale de Lille, BP 48, Cité Scientifique, 59651 Villeneuve d'Ascq and with Équipe Projet ALIEN, INRIA Lille-Nord Europe, France.thierry.floquet@ec-lille.fr presented in [13], [26] show that an appropriate choice of sliding surface, used with the equivalent control required to ensure sliding, can guarantee a bounded motion about the surface in the presence of bounded matched uncertainty and that the use of a relay/switch in the control law is detrimental to performance.

Early contributions in sliding mode control were developed in a framework in which all the system states are available. This may not be realistic for practical engineering problems and has motivated the need for output feedback controllers. A number of algorithms have been developed for robust stabilization of uncertain systems which are based on sliding surfaces and output feedback control schemes [30], [5]. In [30], a geometric condition is developed to guarantee the existence of the sliding surface and the stability of the reduced order sliding motion. Edwards and Spurgeon derived an algorithm [5], [6] which is convenient for practical use. Based on the work in [30], some dynamic feedback sliding mode controllers have been proposed [15], [24]. In all the above output feedback sliding mode control schemes, it is an a priori requirement that the system under consideration is minimum phase and relative degree one and that a particular sub-system must be output feedback stabilisable [6].

Compared with continuous time sliding-mode strategies, the design problem in discrete time is much less mature. Other than early work in [25], much of the literature assumes all states are available [10], [13], [28]. Discrete sliding mode control schemes which have restricted themselves to output measurements alone have often been observer based schemes with or without disturbance estimation [17], [27]. Recent exceptions have been the work in [20] which considers both static and dynamic output feedback problems, and the discrete time versions of certain higher-order sliding-mode control schemes [1], [2].

For continuous time systems, it was shown in [9] that the relative degree condition associated with the solution of the existence problem can be weakened if a classical sliding mode observer is combined with sliding mode exact differentiators to generate additional independent output signals from the available measurements. In [12], it was shown that by using the output signal at the current time instant together with a limited amount of information from previous sample instants, the class of systems for which an output feedback based sliding mode controller can be developed is significantly broadened. It is shown here that if the extended output spans the state zero directions of an invariant zero of the original plant, the invariant zero disappears from the augmented system. The design of the 
sliding surface is formulated as an LMI problem. It is shown that by extending the available outputs, the methodology can be applied to cases where systems experience multiple sensor failures and require reconfigurable control using the remaining available outputs.

The paper is structured as follows. Section 2 presents the problem motivation. The existence problem is considered in Section 3 and a control law to ensure the sliding mode is attained is given in Section 4. A motivational reconfigurable control example, from the class of discrete systems which could not previously be stabilised by output feedback sliding mode control, is presented to illustrate the approach.

\section{Motivation}

Consider the discrete, linear, time invariant state space system representation given below:

$$
\begin{aligned}
x_{k+1} & =A x_{k}+B u_{k} \\
y_{k} & =\left[\left(y_{1}\right)_{k} \ldots\left(y_{p}\right)_{k}\right]^{T}=C x_{k},\left(y_{i}\right)_{k}=C_{i} x_{k}
\end{aligned}
$$

where $x_{k} \in \mathbb{R}^{n}$ is the state vector, $y_{k} \in \mathbb{R}^{p}$ is the output vector and $u_{k} \in \mathbb{R}^{m}$ is the control input. It is assumed that $m \leq p$, the pair $(A, B)$ is controllable and without loss of generality, that $\operatorname{rank}(C)=p$ and that column $\operatorname{rank}(B)=m$.

Consider the development of a control law based on output measurements only which will induce an ideal sliding motion on the surface

$$
s=\left\{x \in \mathbb{R}^{n}: F C x_{k}=0\right\}
$$

for some selected matrix $F \in \mathbb{R}^{m \times p}$. It is well known that for a unique equivalent control to exist, the matrix $F C B \in \mathbb{R}^{m \times m}$ must have full rank. As

$$
\operatorname{rank}(F C B) \leq \min \{\operatorname{rank}(F), \operatorname{rank}(C B)\}
$$

it follows that both $F$ and $C B$ must have full rank. As $F$ is a design parameter, it can be chosen to be full rank. A necessary condition for $F C B$ to be full rank, and thus for solvability of the output feedback sliding mode design problem, thus becomes that $C B$ must have rank $m$. If this rank condition holds and any invariant zeros of the triple $\{A, B, C\}$ lie in the unit disk, then the existence of a matrix $F$ defining the surface (3), which provides a stable sliding motion with a unique equivalent control is determined from the stabilizability by output feedback of a specific, welldefined subsystem of the plant [6].

\section{The Existence Problem}

It will be assumed in this work that the matrix $A$ is invertible. Such a property often occurs in discrete-time linear systems. For instance, consider the continuous time linear time invariant system

$$
\dot{z}(t)=F z(t)+G u(t)
$$

If the system (1)-(2) is the discretized form of the continuous time system (5) under sampling i.e,

$$
A=e^{(F t)}, B=\int_{0}^{T} e^{F t} d \tau G
$$

then it is shown in [22] (page 386) that the state space matrix $A$ is invertible.

Thus, the system (2) can be rewritten as:

$$
\begin{aligned}
& x_{k}=A^{-1}\left(x_{k+1}-B u_{k}\right) \\
& y_{k}=C x_{k}
\end{aligned}
$$

\section{A. Generation of the extended output}

Consider system (1)-(2) without any a priori assumptions relating to either the stability of the invariant zeros or the fulfillment of the matching condition. The main idea here is to construct a matrix $\tilde{C}$ :

$$
\tilde{C}=\left[\begin{array}{c}
C_{1} \\
\vdots \\
C_{1} A^{-\mu_{1}+1} \\
\vdots \\
C_{p} \\
\vdots \\
C_{p} A^{-\mu_{p}+1}
\end{array}\right]
$$

such that $\tilde{C}$ is full $\operatorname{rank}, \operatorname{rank}(\tilde{C} B)=\operatorname{rank}(B)$, and any invariant zeros of the triple $\{A, B, \tilde{C}\}$ lie inside the unit disk. Also, the $\mu_{i}$ are chosen such that $\tilde{p}=\sum_{i=1}^{p} \mu_{i}$ is minimal.

Note that $\tilde{C}=C$ means that the original system is output feedback stabilizable using existing methods (see e.g. [6]).

\section{B. Zeros and Zero directions}

Consider the system in (1)-(2) with $x_{k} \in \mathbb{R}^{n}, u_{k} \in \mathbb{R}^{m}$, $y_{k} \in \mathbb{R}^{p}$, where $A, B, C$ are all real matrices with appropriate dimensions. Then, the number $z$ is defined as the invariant zero of (1)-(2) iff there exists vectors $0 \neq x_{z} \in \mathbb{C}^{n}$ and $u_{z} \in \mathbb{C}^{m}$, such that the triple $z, x_{z}, u_{z}$ satisfies

$$
\left[\begin{array}{cc}
z I-A & B \\
C & 0
\end{array}\right]\left[\begin{array}{l}
x_{z} \\
u_{z}
\end{array}\right]=0
$$

where, $I$ is the identity matrix of dimension $n$. Here, $x_{z}$ is called the state zero direction and $u_{z}$ is called the input zero direction. As seen in [12], it can be shown that any invariant zeros of the triple $\{A, B, \tilde{C}\}$ are amongst the invariant zeros of the triple $\{A, B, C\}$. It can also be shown that if an appropriate choice of extended outputs is available, the invariant zeros from the original triple $(A, B, C)$ may disappear from the augmented system. This is particularly useful if any of the invariant zeros of $(A, B, C)$ are unstable. The choice of augmented outputs to remove transmission zeros is explained in the lemma below.

\section{Lemma 1}

Let $z$ be the invariant zero of the original triple $(A, B, C)$ with zero direction $x_{z}$. Assume the zero direction $x_{z}^{T}$ is spanned by the rows of the augmented output matrix $\tilde{C}$. Then the invariant zero $z$ is not present in the augmented triple $(A, B, \tilde{C})$.

If $z$ is an invariant zero of the original triple with zero direction $x_{z}$, it follows that

$$
C x_{z}=0
$$


and thus $x_{z}$ is in the null space of $C$.

By construction of the augmented output, $x_{z}$ belongs to the range space of $\tilde{C}$. The additional rows of $\tilde{C}$ are constructed by post multiplying particular rows of $\mathrm{C}$ by a full rank matrix of the form $A^{-l}$. It follows that the augmented rows of $\tilde{C}$ lie in the range of $\mathrm{C}$ and thus $\tilde{C} x_{z}$ cannot be identically zero as $x_{z}$ lies in the null space of $C$ by definition. Thus, $z$ is not an invariant zero of $(A, B, \tilde{C})$.

Hence by suitably selecting the augmented outputs such that they span the state zero direction of an unstable invariant zero, it is possible to remove unstable invariant zeros from the augmented system.

\section{Design of the sliding manifold and analysis of the equiv- alent dynamics}

Assume that it is possible to construct a matrix $\tilde{C}$ as defined in (9). Then, the requirements for solvability of the output feedback sliding mode design problem are determined by the triple $\{A, B, \tilde{C}\}$. It remains to find a suitable sliding variable that depends on the available measurements only.

Extend the original outputs as shown below:

$$
\tilde{y}_{k}=\left[\begin{array}{c}
\left(y_{1}\right)_{k} \\
\vdots \\
\left(y_{1}\right)_{k-\mu_{1}+1} \\
\vdots \\
\left(y_{p}\right)_{k} \\
\vdots \\
\left(y_{p}\right)_{k-\mu_{p}+1}
\end{array}\right]
$$

From the system (7)-(8), it can be computed that:

$$
\begin{aligned}
y_{k} & =C x_{k} \\
y_{k-1} & =C A^{-1}\left(x_{k}-B u_{k-1}\right) \\
y_{k-2} & =C A^{-2} x_{k}-C A^{-2} B u_{k-1}-C A^{-1} B u_{k-2} \\
& \vdots \\
y_{k-j} & =C A^{-j} x_{k}-C A^{-j} B u_{k-1}-\cdots-C A^{-1} B u_{k-j} \\
& =C A^{-j} x_{k}-\sum_{l=1}^{j} C A^{-l} B u_{k-j+l-1}
\end{aligned}
$$

Thus, the extended output matrix $\tilde{y}$ can be expressed as follows:

$$
\tilde{y}_{k}=\tilde{C} x_{k}-M\left[\begin{array}{c}
\bar{u}_{1} \\
\bar{u}_{2} \\
\vdots \\
\bar{u}_{p}
\end{array}\right]
$$

with, for $i=1, \ldots, p$ :

$$
\begin{gathered}
\bar{u}_{i}=\left[\begin{array}{c}
u_{k-1} \\
u_{k-2} \\
\vdots \\
u_{k-\mu_{i}+1}
\end{array}\right] \\
\mathbf{M}=\operatorname{diag}\left\{\mathbf{M}_{1}, \ldots, \mathbf{M}_{p}\right\}
\end{gathered}
$$

$$
\mathbf{M}_{i}=\left(\begin{array}{ccccc}
0 & 0 & \ldots & \ldots & 0 \\
C_{i} A^{-1} B & 0 & \ldots & \ldots & 0 \\
C_{i} A^{-2} B & C_{i} A^{-1} B & \ddots & & \vdots \\
\vdots & & \ddots & 0 & \vdots \\
\vdots & & & C_{i} A^{-1} B & 0 \\
C A^{-\mu_{i}+1} B & \ldots & \ldots & C_{i} A^{-2} B & C_{i} A^{-1} B
\end{array}\right)
$$

where $\mathbf{M}_{i} \in \mathbb{R}^{\mu_{i} \times\left(\mu_{i}-1\right)}$. If the outputs are extended such that $\operatorname{rank}(\tilde{C})$ is $n$ (i.e when $\tilde{C}$ is invertible), it can be seen from (11) that it is possible to write the state as a function of $\tilde{y}$ (the current and the past outputs) and $u_{i}$ (the past inputs). In this case the transmission zeros can always be removed from the augmented system and the controller design is performed as a state feedback design using the estimates of the states. The following class of sliding manifolds, that only depend on known variables, can then be defined

$$
\begin{aligned}
s_{k} & =F \tilde{y}_{k}+F M\left[\begin{array}{c}
\bar{u}_{1} \\
\bar{u}_{2} \\
\vdots \\
\bar{u}_{p}
\end{array}\right] \\
& =F \tilde{C} x_{k}
\end{aligned}
$$

where $F \in^{m \times \tilde{p}}$ is a design parameter.

To analyse the stability of the resulting sliding motion, it is now convenient to introduce a coordinate transformation to the usual regular form, making the final $\tilde{p}$ states of the system depend directly on the extended outputs [6]:

$$
\begin{aligned}
A & =\left[\begin{array}{ll}
A_{11} & A_{12} \\
A_{21} & A_{22}
\end{array}\right] B=\left[\begin{array}{c}
0 \\
B_{2}
\end{array}\right] \tilde{C}=\left[\begin{array}{ll}
0 & T
\end{array}\right] \\
x & =\left[\begin{array}{l}
\bar{x}_{1} \\
\bar{x}_{2}
\end{array}\right]
\end{aligned}
$$

where $T \in \mathbb{R}^{\tilde{p} \times \tilde{p}}$ is an orthogonal matrix, $A_{11} \in \mathbb{R}^{(n-m) \times(n-m)}$ and the remaining sub-blocks in the system matrix are partitioned accordingly. The corresponding switching surface parameter is given by

$$
\begin{aligned}
& \stackrel{\tilde{p}-m}{\leftrightarrow} \stackrel{m}{\leftrightarrow} \\
& {\left[\begin{array}{ll}
F_{1} & F_{2}
\end{array}\right]=F T}
\end{aligned}
$$

where $T$ is the matrix from equation (14). As a result

$$
F \tilde{C}=\left[\begin{array}{ll}
F_{1} C_{f} & F_{2}
\end{array}\right]
$$

where

$$
C_{f}=\left[\begin{array}{ll}
0_{(\tilde{p}-m) \times(n-\tilde{p})} & I_{(\tilde{p}-m)}
\end{array}\right]
$$

Therefore $F \tilde{C} B=F_{2} B_{2}$ and the square matrix $F_{2}$ is nonsingular. The canonical form in (14) can be viewed as a special case of the regular form normally used in sliding mode controller design, and thus the reduced-order sliding motion is governed by a free motion with system matrix

$$
A_{11}^{s}=A_{11}-A_{12} F_{2}^{-1} F_{1} C_{f}
$$

which must therefore be stable. If $K \in \mathbb{R}^{m \times(\tilde{p}-m)}$ is defined as $K=F_{2}^{-1} F_{1}$ then

$$
A_{11}^{s}=A_{11}-A_{12} K C_{f}
$$


and the problem of hyperplane design is equivalent to a static output feedback problem for the system $\left(A_{11}, A_{12}, C_{f}\right)$. In order to utilize the existing literature it is necessary that the pair $\left(A_{11}, A_{12}\right)$ is controllable and $\left(A_{11}, C_{f}\right)$ is observable. The former is ensured as $(A, B)$ is controllable. The observability of $\left(A_{11}, C_{f}\right)$, is not so straightforward, but can be investigated by considering the canonical form below.

\section{Lemma 2}

Let $(A, B, \tilde{C})$ be a linear system with $\tilde{p}>m$ and $\operatorname{rank}(\tilde{C} B)=m$. Then a change of coordinates exists so that the system triple with respect to the new coordinates has the following structure:

- The system matrix can be written as

$$
A=\left[\begin{array}{ll}
A_{11} & A_{12} \\
A_{21} & A_{22}
\end{array}\right]
$$

where $A_{11} \in \mathbb{R}^{(n-m) \times(n-m)}$ and the sub-block $A_{11}$ when partitioned has the structure

$$
A_{11}=\left[\begin{array}{cc|c}
A_{11}^{o} & A_{12}^{o} & A_{12}^{m} \\
0 & A_{22}^{o} & \\
\hline 0 & A_{21}^{o} & A_{22}^{m}
\end{array}\right]
$$

where $A_{11}^{o} \in \mathbb{R}^{r \times r}, A_{22}^{o} \in \mathbb{R}^{(n-\tilde{p}-r) \times(n-\tilde{p}-r)}$ and $A_{21}^{o} \in$ $\mathbb{R}^{(\tilde{p}-m) \times(n-\tilde{p}-r)}$ for some $r \geq 0$ and the pair $\left(A_{22}^{o}, A_{21}^{o}\right)$ is completely observable.

- The input distribution matrix $B$ and the output distribution matrix $\tilde{C}$ have the structure in (14).

For a proof and a constructive algorithm to obtain this canonical form see [5].

In the case where $r>0$, the intention is to construct a new system $\left(\tilde{A}_{11}, \tilde{B}_{1}, \tilde{C}_{f}\right)$ which is both controllable and observable with the property that

$$
\lambda\left(A_{11}^{s}\right)=\lambda\left(A_{11}^{o}\right) \cup \lambda\left(\tilde{A}_{11}-\tilde{B}_{1} K \tilde{C}_{f}\right) .
$$

To this end, as in [5], partition the matrices $A_{12}$ and $A_{12}^{m}$ as

$$
A_{12}=\left[\begin{array}{l}
A_{121} \\
A_{122}
\end{array}\right] \quad \text { and } \quad A_{12}^{m}=\left[\begin{array}{c}
A_{121}^{m} \\
A_{122}^{m}
\end{array}\right]
$$

where $A_{122} \in \mathbb{R}^{(n-m-r) \times m}$ and $A_{122}^{m} \in \mathbb{R}^{(n-\tilde{p}-r) \times(\tilde{p}-m)}$ and form a new sub-system represented by the triple $\left(\tilde{A}_{11}, A_{122}, \tilde{C}_{f}\right)$ where

$$
\begin{aligned}
\tilde{A}_{11} & =\left[\begin{array}{ll}
A_{22}^{o} & A_{122}^{m} \\
A_{21}^{o} & A_{22}^{m}
\end{array}\right] \\
\tilde{C}_{f} & =\left[\begin{array}{ll}
0_{(\tilde{p}-m) \times(n-\tilde{p}-r)} & I_{(\tilde{p}-m)}
\end{array}\right]
\end{aligned}
$$

It follows that the spectrum of $A_{11}^{S}$ decomposes as

$$
\lambda\left(A_{11}-A_{12} K C_{f}\right)=\lambda\left(A_{11}^{o}\right) \cup \lambda\left(\tilde{A}_{11}-A_{122} K \tilde{C}_{f}\right)
$$

\section{Lemma 3 [5]}

The spectrum of $A_{11}^{o}$ represents the invariant zeros of $(A, B, \tilde{C})$.

It follows directly that for a stable sliding motion, the invariant zeros of the system $(A, B, \tilde{C})$ must lie inside the unit disk and the triple $\left(\tilde{A}_{11}, A_{122}, \tilde{C}_{f}\right)$ must be stabilisable with respect to output feedback.
The matrix $A_{122}$ is not necessarily full rank. Suppose $\operatorname{rank}\left(A_{122}\right)=m^{\prime}$ then, as in [5], it is possible to construct a matrix of elementary column operations $T_{m^{\prime}} \in \mathbb{R}^{m \times m}$ such that

$$
A_{122} T_{m^{\prime}}=\left[\begin{array}{cc}
\tilde{B}_{1} & 0
\end{array}\right]
$$

where $\tilde{B}_{1} \in \mathbb{R}^{(n-m-r) \times m^{\prime}}$ and is of full rank. If $K_{m^{\prime}}=T_{m^{\prime}}^{-1} K$ and $K_{m^{\prime}}$ is partitioned compatibly as

$$
K_{m^{\prime}}=\left[\begin{array}{l}
K_{1} \\
K_{2}
\end{array}\right] \begin{aligned}
& \uparrow m^{\prime} \\
& \uparrow m-m^{\prime}
\end{aligned}
$$

then

$$
\begin{aligned}
\tilde{A}_{11}-A_{122} K \tilde{C}_{f} & =\tilde{A}_{11}-\left[\begin{array}{cc}
\tilde{B}_{1} & 0
\end{array}\right] K_{m^{\prime}} \tilde{C}_{f} \\
& =\tilde{A}_{11}-\tilde{B}_{1} K_{1} \tilde{C}_{f}
\end{aligned}
$$

and $\left(\tilde{A}_{11}, A_{122}, \tilde{C}_{f}\right)$ is stabilizable by output feedback if and only if $\left(\tilde{A}_{11}, \tilde{B}_{1}, \tilde{C}_{f}\right)$ is stabilizable by output feedback. It follows that:

\section{Lemma 4}

The pair $\left(\tilde{A}_{11}, \tilde{B}_{1}\right)$ is completely controllable and $\left(\tilde{A}_{11}, \tilde{C}_{f}\right)$ is completely observable.

To design the sliding surface consider the method for design of a static output feedback gain as given in [18]. The technique in [18] introduces slack variables to decouple the Lyapunov matrix and the static output feedback gain. With the additional slack variables and a chosen state space variable, an LMI problem is solved to obtain the static output feedback controller. The following theorem is required to formulate the design of the sliding surface using this method.

\section{Theorem 1}

A static output feedback gain $K$ is stabilizing if and only if there exists a positive definite matrix $P=P^{T}>0$ in $\mathbb{R}^{n \times n}$, non singular matrices $G \in \mathbb{R}^{m \times m}$ and $E_{4} \in \mathbb{R}^{n \times n}$, a non-null matrices $E_{1} \in \mathbb{R}^{n \times n}$ and $L \in \mathbb{R}^{m \times p}$ and arbitrary matrices $E_{2} \in \mathbb{R}^{n \times n}, E_{3} \in \mathbb{R}^{m \times n}$ such that the following LMI:

$$
\left.\begin{array}{cc}
E_{1} A_{o}+A_{o}^{T} E_{1}^{T}-P & * \\
E_{2} A_{o} & -P \\
E_{3} A_{o}+A_{122}^{T} E_{1}^{T}+\left(L \tilde{C}_{f}-G K_{o}\right) & A_{122}^{T} E_{2}^{T} \\
E_{4} A_{o}-E_{1}^{T} & P-E_{2}^{T} \\
* & * \\
* & * \\
E_{3} A_{122}+A_{122}^{T} E_{3}^{T}-\left(G+G^{T}\right) & * \\
E_{4} A_{122}-E_{3}^{T} & -E_{4}-E_{4}^{T}
\end{array}\right]<0
$$

is feasible for a given state feedback gain $K_{o}$ that stabilizes the pair $\left(\tilde{A}_{11}, A_{122}\right)$ with the static output feedback gain given by

$$
K=G^{-1} L
$$

The matrix $A_{o}$ here is defined as $A_{o}=\tilde{A}_{11}+A_{122} K_{o}$. For a conclusive proof for the above theorem refer to [18]. 


\section{Reachability Problem}

It is necessary to force $s(k)$ to zero to ensure the sliding dynamics are reached. Here the control selected has two components and is of the form:

$$
u_{k}=K_{y} \tilde{y}_{k}-\left(\Phi-A_{22}\right) s_{k}
$$

Consider

$$
T_{s}=\left[\begin{array}{cc}
I_{n-m} & 0 \\
F_{1} C_{f} & F_{2}
\end{array}\right]
$$

with the sub matrices of $T_{s}$ conformably partitioned. The matrix $T_{s}$ is nonsingular since by construction $F_{2}$ is nonsingular. Let

$$
\left[\begin{array}{c}
\left(x_{1}\right)_{k} \\
s_{k}
\end{array}\right]=T_{s}\left[\begin{array}{l}
\left(x_{1}\right)_{k} \\
\left(x_{2}\right)_{k}
\end{array}\right]
$$

Then with the new coordinates the nominal system can be written as:

$$
\begin{array}{r}
\left(x_{1}\right)_{k+1}=\bar{A}_{11}\left(x_{1}\right)_{k}+\bar{A}_{12} s_{k} \\
s_{k+1}=F_{2} \bar{A}_{21}\left(x_{1}\right)_{k}+F_{2} \bar{A}_{22} F_{2}^{-1} s_{k}+\Lambda u_{k}
\end{array}
$$

with $\bar{A}_{11}=A_{11}-A_{12} M, \bar{A}_{12}=A_{12} F_{2}^{-1}, \bar{A}_{21}=M \bar{A}_{11}+$ $A_{21}-A_{22} M$ and $\bar{A}_{22}=M \bar{A}_{12}+A_{22}$, with $M=F_{2}^{-1} F_{1} C_{f}$ and $\Lambda=F_{2} B_{2}$.

The first component of (24) is developed for stabilising the system (27) and is calculated by using the algorithm given in [18]. The second component of the control law is used for shaping the reaching response of the plant and is a design choice. The control law chosen is similar to the linear control law given in [20]. The control law here does not use any dynamics in the sense that there is no explicit estimation of states or an explicit compensator to achieve control. Thus the design can be considered as a static output feedback control law achieved with the available signals.

\section{Motivational Example}

Consider the example of an aircraft from [6] given below. The plant model represents the fifth-order lateral dynamics of an L-1011 fixed wing aircraft, with the actuator dynamics removed. The plant system triple is given by:

$$
\begin{aligned}
& A=\left[\begin{array}{ccccc}
0 & 0 & 1 & 0 & 0 \\
0 & -.154 & -.0042 & 1.54 & 0 \\
0 & .249 & -1.000 & -5.20 & 0 \\
0.0386 & -0.996 & -0.0003 & -.117 & 0 \\
0 & 0.500 & 0 & 0 & -0.5
\end{array}\right] \\
& B=\left[\begin{array}{cc}
0 & 0 \\
-0.744 & -0.032 \\
0.337 & -1.1200 \\
0.02 & 0 \\
0 & 0
\end{array}\right] C=\left[\begin{array}{llllc}
0 & 1 & 0 & 0 & -1 \\
0 & 0 & 1 & 0 & 0 \\
0 & 0 & 0 & 1 & 0 \\
1 & 0 & 0 & 0 & 0
\end{array}\right]
\end{aligned}
$$

The plant states are:

$$
x=\left[\begin{array}{cc}
\phi & \text { bank angle }(\mathrm{rad}) \\
r & \text { yaw rate }(\mathrm{rad} / \mathrm{sec}) \\
p & \text { roll rate }(\mathrm{rad} / \mathrm{sec}) \\
\beta & \text { side slip angle }(\mathrm{rad}) \\
x_{5} & \text { washed-out filter state }
\end{array}\right]
$$

with the inputs being

$$
u=\left[\begin{array}{ll}
\delta_{r} & \text { rudder deflection }(\mathrm{rad}) \\
\delta_{a} & \text { aileron deflection }(\mathrm{rad})
\end{array}\right]
$$

and the outputs

$$
y=\left[\begin{array}{cc}
r_{w o} & \text { washed out yaw rate }(\mathrm{rad}) \\
p \text { roll rate }(\mathrm{rad} / \mathrm{sec}) \\
\beta \text { sideslip angle }(\mathrm{rad}) \\
\phi \text { bank angle }(\mathrm{rad})
\end{array}\right]
$$

The sampling rate chosen for discretizing the plant was $t=.1 s$. Assume that the aircraft has lost the side slip angle and roll rate.The invariant zeros for the resulting plant triple $(A, B, C)$ are at $[1.00 .9929-.9671]$ and thus a stable sliding surface cannot be designed using the remaining two outputs. By augmenting the bank angle and the washed out yaw rate once, it is found that the invariant zeros are removed from the system with the augmented outputs. The augmented matrix $\tilde{C}$ in this case is given as

$$
\tilde{C}=\left[\begin{array}{ccccc}
0 & 1.0000 & 0 & 0 & -1.0000 \\
0.0003 & 1.0593 & 0.0004 & -0.1595 & -1.0513 \\
1.0000 & 0 & 0 & 0 & 0 \\
1.0000 & 0.0004 & -0.1052 & -0.0270 & 0
\end{array}\right]
$$

Here the orthogonal transformation matrix $T$ is

$$
T=\left[\begin{array}{cccc}
-0.5345 & 0.4990 & -0.0132 & -0.6820 \\
0.4980 & -0.4661 & -0.0185 & -0.7310 \\
0.4973 & 0.5349 & -0.6829 & 0.0149 \\
0.4680 & 0.4975 & 0.7302 & -0.0169
\end{array}\right]
$$

and the subsystem $\left(\tilde{A}_{11}, A_{122}, \tilde{C}_{f}\right)$ obtained for the sliding surface design is

$$
\begin{gathered}
\tilde{A}_{11}=\left[\begin{array}{ccc}
0.9933 & -0.0328 & 0.0307 \\
0.0056 & 1.1861 & -0.1149 \\
-0.0053 & 0.2090 & 0.8684
\end{array}\right] \\
A_{122}=\left[\begin{array}{cc}
0.0050 & -0.0414 \\
-1.2966 & 0.0211 \\
-1.3871 & 0.0344
\end{array}\right] \tilde{C}_{f}=\left[\begin{array}{lll}
0 & 1 & 0 \\
0 & 0 & 1
\end{array}\right]
\end{gathered}
$$

The initial state feedback gain $K_{o}$ is obtained by pole placement and the poles are placed at $\left[\begin{array}{lll}.85 & .975 & .9\end{array}\right]$. Using the algorithm given in [18], the gain $K$ for the reduced order subsystem $\left(\tilde{A}_{11}, A_{122}, \tilde{C}_{f}\right)$ is calculated as

$$
K=\left[\begin{array}{cc}
0.2838 & 0.2682 \\
-0.3432 & -6.8063
\end{array}\right]
$$

with the $\lambda\left(\tilde{A}_{11}-A_{122} K \tilde{C}_{f}\right)$ at $[0.9849+0.0281 i 0.9849-$ $0.0281 i 0.0969]$. The output feedback gain $K_{y}$ is obtained by first obtaining a state feedback gain by placing the poles at [.98 .975 .96 .985 .95$]$. Using the algorithm in [18] modified for the system given in (27), the static output feedback gain is calculated as:

$$
K_{y}=\left[\begin{array}{cccc}
-5.2236 & 10.0550 & -1.7262 & 0.0532 \\
7.4514 & -15.1651 & 2.1197 & 1.2640
\end{array}\right]
$$



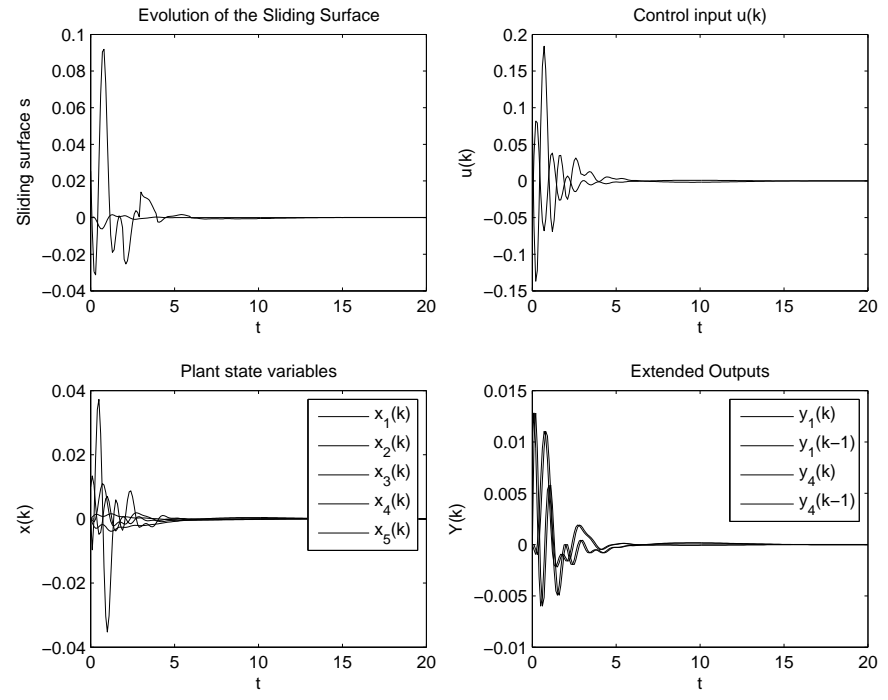

Fig. 1. Response of the states $x_{k}$, Control Input $u_{k}$, sliding surface $s_{k}$, and augmented plant output $\tilde{y}_{k}$

Here $\Phi$ is chosen as $.95 I_{m}$, where $I_{m}$ is the identity matrix of dimension $m \times m$. The simulations were performed with the control law given in (24) and with the initial conditions $\left[\begin{array}{lllll}0 & .01 & 0 & 0 & 0\end{array}\right]$. The simulation results are shown below. Recall that this control law is performing system reconfiguration following the loss of two sensors. The key design requirement is that the system response remain stable given only measurements from the remaining sensors. In such circumstances one would not expect to be able to match the performance achievable from the complete set of measurements.

\section{CONCLUSIONS}

A static output feedback sliding mode-type control has been developed for discrete systems which employs information from both the previous and current output measurements. It has been shown that the invariant zeros of the original system can be removed from an augmented system if the minimal set of current and past outputs used to determine the extended signal span the state input direction associated with the invariant zero. The sliding surface design was performed using Linear Matrix Inequalities. It has been shown that the theory is applicable for systems in the presence of sensor failure by considering a particular aircraft problem. It is hypothesized that the methodology presented in this paper provides a useful core for developing reconfigurable control schemes for discrete time implementation.

\section{REFERENCES}

[1] G. Bartolini, A. Pisano and E. Usai, Digital second-order sliding mode control for uncertain nonlinear systems, Automatica, 37, 1371-1377, 2001.

[2] G. Bartolini, A. Pisano and E. Usai, Digital sliding mode control with $O\left(T^{3}\right)$ accuracy, Proceedings of the 6th IEEE International Workshop on VSS, Koolongata, Australia, 2000.

[3] A. Bartoszewicz, Comments on discrete-time variable structure control systems, IEEE Transactions on Industrial Electronics, 43, 235-238, 1996.
[4] C. L. Chen, K. C. Lin and M. J. Jang, On chattering free output feedback sliding mode design for MIMO linear systems, Proceedings of the 16th IFAC World Congress, Prague, July 3-8, 2005.

[5] C. Edwards and S. K. Spurgeon, Sliding mode stabilisation of uncertain systems using only output information, International Journal of Control, 62, 1129-1144, 1995.

[6] C. Edwards and S. K. Spurgeon, Sliding Mode Control: theory and applications, Taylor and Francis, 1998.

[7] S. V. Emel'yanov, S. K. Korovin, and L. V. Levantovsky, Higher Order Sliding Modes in the Binary Control System, Soviet Physics, 31, No 4, 291-293, 1986.

[8] T. Floquet, C. Edwards and S. K. Spurgeon, On Sliding Mode Observers for Systems with Unknown Inputs, International Journal of Adaptive Control and Signal Processing, 21, No 8-9, 638-656, 2007.

[9] T. Floquet, S. K. Spurgeon and C. Edwards, An output feedback sliding mode control strategy for MIMO systems of arbitrary relative degree, IEEE Conf. on Decision and Control, New-Orleans, USA, 2007.

[10] K. Furuta and Y. Pan, Variable structure control with sliding sector, Automatica, 6, 211-228, 2000.

[11] W. Gao, Y. Wang and A. Homaifa, Discrete-time variable structure control systems, IEEE Transactions on Industrial Electronics, 42, 117122, 1995.

[12] S. Govindaswamy T. Floquet and S.K. Spurgeon, On output sampling based sliding mode control for discrete time systems, IEEE Conf. on Decision and Control, Cancun, Mexico, 2008.

[13] S. Hui and S. H. Zak, On discrete-time variable structure sliding mode control, Systems and Control Letters, 38, 283-288, 1999.

[14] S. Janardhanan and B. Bandyopadhyay, Multirate Output Feedback based Robust Quasi-Sliding Mode Control of Discrete time systems, IEEE Transactions on Automatic Control, 52, 499-503, 2007.

[15] C. M. Kwan, Further results on variable output feedback controllers, IEEE Transactions on Automatic Control, 46, 1505-1508, 2001.

[16] N. O. Lai, C. Edwards and S. K. Spurgeon, On Output Tracking Using Dynamic Output Feedback Discrete-Time Sliding-Mode Controllers, IEEE Transactions on Automatic Control, 52, 1975-1981, 2007.

[17] S. M. Lee and B. H. Lee, A discrete-time sliding mode controller and observer with computation delay, Control Engineering Practice, 7, 2943-2955, 1999.

[18] D. Mehdi, E. K. Boukas and O. Bachelier, Static output feedback design for uncertain linear systems, IMA Journal of Mathematical Control and Information, 21,1-13, 2004.

[19] C. Milosavljevic, Discrete-time VSS, in Variable Structure Systems: From Principles to Implementation, Chapter 5, A. Sabanovic, L. Fridman, S. K. Spurgeon (eds). Institution of Electrical Engineers: U.K., 2004.

[20] G. Monsees, Discrete-time sliding mode control, Ph.D. Thesis, Delft University of Technology, The Netherlands, 2002.

[21] W. Perruquetti and J.-P. Barbot (Editors), Sliding Mode Control in Engineering, Marcel Dekker, 2002.

[22] W. J. Rugh, Linear Systems Theory, Prentice Hall: New Jersey, 1996.

[23] S. Z. Sapturk, Y. Istefanopulous and O. Kaynak, On the stability of discrete-time sliding mode control systems, IEEE Transactions on Automatic Control, 32, 930-932, 1987.

[24] K. K. Shyu, Y. W. Tsai and C. K. Lai, A dynamic output feedback controller for mismatched uncertain variable systems, Automatica, 37, 775-779, 2001.

[25] H. Sira-Ramirez, Nonlinear discrete variable structure systems in quasi-sliding mode, International Journal of Control, 54, 1171-1187, 1991.

[26] S. K. Spurgeon, Hyperplane design techniques for discrete-time variable structure control systems, International Journal of Control, 55, 445-456, 1992.

[27] C. Y. Tang and E. Misawa, Discrete variable structure control for linear multivariable systems, Journal of Dynamic Systems, Measurement, and Control, 122, 783-792, 2000.

[28] C. Y. Tang and E. Misawa, Sliding surface design for discrete VSS using LQR technique with a preset eigenvalue, Systems and Control Letters, 45, 1-7, 2002.

[29] V. I. Utkin, Sliding Modes in Control Optimization, Springer: Berlin, 1992.

[30] S. H. Zak and S. Hui, On variable structure output feedback controllers for uncertain dynamic systems, IEEE Transactions on Automatic Control, 38, 1509-1512, 1993. 